\title{
Uma nova perspectiva da utilização do DOSVOX no tratamento das deficiências cognitivas
}

\section{A new perspective on the use of DOSVOX in the treatment of cognitive disabilities}

\section{Flavia Ernesto de Oliveira da Silva Alves}

Aluna Ouvinte Doutoranda do Programa de Pós-Graduação em História das Ciências e das Técnicas e Epistemologia (HCTE), Universidade Federal do Rio de Janeiro (UFRJ). flaviaernesto@gmail.com

orcid.org/0000-0001-7111-9234

\section{Denise Alvares de Oliveira}

Aluna Ouvinte Mestranda do Programa de Pós-Graduação em História das Ciências e das Técnicas e Epistemologia (HCTE), Universidade Federal do Rio de Janeiro (UFRJ). denisecaoliveira@hotmail.com orcid.org/0000-0003-1954-1889

Resumo. Este trabalho apresenta a experiência na aplicação de tecnologia utilizada para pessoas com deficiências visuais em uma nova abordagem. $\mathrm{Na}$ seção de introdução são apresentadas as motivações que levaram ao seu desenvolvimento. Nas seções seguintes, além de apresentar as teorias que encadearam a realização da atividade, bem como o público alvo em questão, é apresentada uma reflexão sobre a complexidade do pensamento, para a contextualização e definição da proposta: a aplicação do Sistema DOSVOX como recurso para o tratamento de pessoas comdeficiências cognitivas. Em seguida é apresentada a teoria ator-rede que permite a conexão entre os diversos atores desse contexto. Na seção resultados são pontuadas as principais observações acerca do trabalho.

Palavras-chave: AVC. Cognição. DOSVOX.

Abstract.This work presents the experience in the application of technology used for people with visual impairments in a new approach. The introduction section presents the motivations that led to its development. In the following sections, in addition to presenting the theories that linked the performance of the activity, as well as the target audience in question, a reflection on the complexity of thought is presented, for the context and definition of the proposal: the application of the DOSVOX System as a resource for the treatment of people with cognitive 
disabilities. Then, the actor-network theory that allows the connection between the different actors in this context is presented. The results section highlights the main observations about the work.

Keywords: Stroke. Cognition. DOXVOX.

Recebido: 01/10/2017 Aceito: 27/10/2017 Publicado: 06/11/2017

\section{Introdução}

No decorrer da disciplina Deftas (Deficiência, Tecnologia Assistiva e Sociedade - 1), onde foram apresentados diversos sistemas voltados ao tratamento de pessoas com deficiência, percebeu-se a possibilidade da utilização do Sistema DOSVOX, criado para pessoas com deficiência visual, ser utilizado sob uma nova perspectiva, atendendo também às pessoas com déficits cognitivos. Essa atividade foi construída a partir da parceria formada entre duas profissionais: uma Terapeuta Ocupacional e uma Analista de Sistemas e Pedagoga, que juntas iniciaram a elaboração de jogos terapêuticos voltados as necessidades do tratamento das alterações cognitivas relacionadas ao Acidente Vascular Cerebral (AVC)

\section{Percurso Metodológico}

\subsection{Acidente Vascular Cerebral (AVC)}

O Acidente Vascular Cerebral (AVC)descreve uma variedade de distúrbios caracterizados pelo início súbito de déficits neurológicos causados por lesão cerebral. A lesão vascular interrompe fluxo sanguíneo, limitando o suprimento das células e induzindo a morte ou infarto do tecido cerebral. (TROMBLY; RADOMSKI,2005). O mecanismo, a localização e a extensão da lesão determinam os sintomas e o prognóstico do paciente. Dentre as disfunções que se apresentam e que interferem na recuperação da pessoa afetada estão as alterações cognitivas. (TROMBLY;RADOMSKI,2005)

\subsection{Cognição}

O termo cognição reflete o empreendimento mental relacionado a absorver informação, pensamento e ação dirigida a um objetivo. Esta consiste em uma hierarquia interativa que inclui as capacidades de orientação, atenção, memória, raciocínio, formação de conceitos e solução de problemas. Os objetivos do tratamento e os recursos utilizados para abordar os déficits devem ter um propósito claro e ser significativos para o paciente (TROMBLY; RADOMSKI,2005). Dentre os recursos possíveis que visam favorecer a recuperação da pessoa pós $\mathrm{AVC}$, temos a 
tecnologia assistiva / computação eletrônica na forma de jogos terapêuticos, os quais devem atender individualmente as necessidades do paciente emquestão, favorecendo o seu desempenho ocupacional nas atividades cotidianas. (TROMBLY\&RADOMSKI,2005).

\subsection{O Raciocinio}

Pensar em uma atividade que seja diferente do seu propósito inicial, nos remete à articulação entre novas e diferentes possibilidades, ocorrendo intrinsecamente um processo de reformulação do pensamento. (MORIN, 2005). A proposta deste artigo contempla uma reformulação de ideiasao descortinar uma nova possibilidade de utilização para um jogo, que traz consigo inúmeras possibilidades, e em corolário, propor a utilização sob uma nova vertente. É bem verdade, que existe uma enorme diversidade de softwares e aplicativos no mercado que foram produzidos para serem utilizados com deficientes visuais, deficientes auditivos, deficientes cognitivos, etc. O que está sendo apresentado nada mais é do que uma nova roupagem para uma tecnologia criada em princípio para outro fim.

\subsection{Tecnologia Assistiva}

A tecnologia criada para apoiar pessoas com deficiência é chamada Tecnologia Assistiva (TA) - um termo utilizado para identificar todo o arsenal de recursos e serviços que contribuem para proporcionar ou ampliar habilidades funcionais de pessoas com deficiência e consequentemente promover vida independente e inclusão (BERSCH, 2006).Em um sentido mais amplo, a TA tem por objetivo proporcionar à pessoa com deficiência uma maior independência, qualidade de vida, inclusão social, ampliar a comunicação, mobilidade, habilidades de seu aprendizado e trabalho, permitindo ao deficiente a diminuição de sua deficiência. "Para as pessoas sem deficiência a tecnologia torna as coisas mais fáceis. Para as pessoas com deficiência, a tecnologia torna as coisas possíveis". (RADABAUGH, 1993).

\section{A Atividade em Ação}

\subsection{O Sistema DOSVOX}

No site do NCE é possível encontrar a definição do DOSVOX como sendo: "um sistema computacional, baseado no uso intensivo de síntese de voz, desenvolvido pelo Instituto Tércio Paciti (antigo Núcleo de Computação Eletrônica (NCE) da Universidade Federal do Rio de Janeiro (UFRJ), que se destina a facilitar o acesso de 
deficientes visuais a microcomputadores. Através de seu uso é possível observar um aumento muito significativo no índice de independência e motivação das pessoas com deficiência visual, tanto no estudo, trabalho ou interaçãocom outras pessoas. Atualmente o projeto conta com mais de 80.000 usuários espalhados pelo Brasil, Portugal e América Latina."A ideia de criação doDOSVOX surgiua partir da necessidade de Marcelo Pimentel, aluno do Curso de Informática da UFRJ em 1992, em estudar matérias que favorecessem o uso do computador. Na época, em 1993, não existia ainda nenhum sistema com síntese de voz para língua portuguesa disponível para uso em microcomputadores IBM PC, que eram aqueles utilizados por todos os alunos de informática na UFRJ . Desse modo o Professor Antônio Borges, informático da UFRJ e Doutor em Engenharia de Sistemas e Computação criou um sistema que fizesse o computador interagir com o usuário através da voz, pois entendeu a importância de favorecer o aluno no processo de inclusão.

"O Dosvox constitui-se em um conjunto de programas que permite a acessibilidade digital, através de um sintetizador de voz em português. Como o sistema lê e digitaliza o som em português, o diálogo homem/máquina é feito de forma simples e sem "jargões". Esse programa também utiliza padrões internacionais de computação, podendo ler dados e textos gerados por programas e sistemas de uso comum em Informática". (FONSECA,2012,p.32)

Desde a sua idealização e início de sua criação em 1993, muitos pesquisadores em diferentes áreas tem encontrado no DOSVOX uma possibilidade de utilização como proposta de inserção da pessoa com deficiência visual nos diferentes segmentos da sociedade.

\subsection{O JogaVOX}

O JogaVOX é um editor de jogos educacionais contido no DOSVOX, cuja proposta central é possibilitar o desenvolvimento de jogos, permitindo a criação de estratégias a serem usadas na abordagem proposta. Ele oferece uma simplicidade operacional muito grande, principalmente se comparado a outros softwares com o mesmo fim, o que possibilita que pessoas possam desenvolver seus próprios jogos através do uso dessa formidável ferramenta.

(CUNHA,2012) nos diz que o JogaVOX pode ser visto como uma ferramenta computacional que torna viável aos professores que trabalham com deficientes visuais desenvolvendo jogos, sem que houvesse necessidade de conhecimentos técnicos computacionais, pois seu objetivo principal deve ser a valorização de atividades que sejam contextualizadas com a realidade da pessoa deficiente visual que estará jogando.

A ideia de criação deste artigo surgiu a partir da apresentação do DOSVOX aos alunos da disciplina DEFTAS 1, quando foi solicitada a elaboração de jogos voltados às pessoas com deficiência visual, editando jogos educacionais na 
modalidade JogaVOX. A construção dos jogos propostos trouxe a percepção de que outras modalidades de jogos poderiam ser criadas, estendendo os benefícios à outras pessoas com deficiências. Tratando-se de uma turma heterogênea, com experiências profissionais distintas, percebeu-se que a associação

dos esforços e competências promovia os melhores resultados. Foi assim que a experiência da Terapeuta Ocupacional no tratamento de pessoas com deficiências se somou à experiência profissional Professora de Informática e Estudante de Pedagogia. Nesta perspectiva, os jogos foram planejados, construídos e aplicados pela Terapeuta Ocupacional no atendimento de pacientes com disfunções neurológicas e déficits cognitivos em tratamento em Centro de Reabilitação. Cabe ressaltar que alguns jogos que acompanham o programa JogaVOX foram também utilizados no decorrer da atividade.

Foram pensadas e organizadas diferentes propostas, sempre tendo como pensamento a necessidade cognitiva que cada paciente precisa explorar: memorização, sequência lógica, através de diferentes formas de desafios que são inseridos como proposta motivadora para reinserção dos pacientes na sociedade. Na Figura 1 é apresentado o exemplo de um Jogo, onde o paciente é convidado a reconhecer a partir de um determinado som, que instrumento o reproduz, com isso trabalhar a percepção auditiva e a memória de maneira lúdica e criativa.

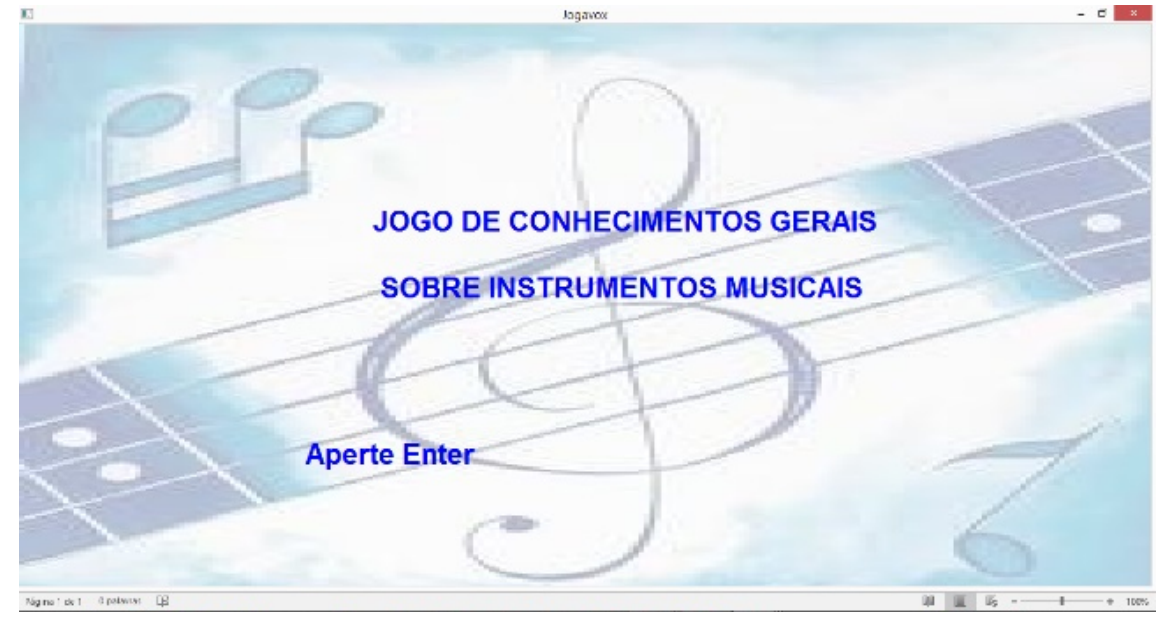

Figura 1. Jogo dos Instrumentos

$\mathrm{Na}$ Figura 2é apresentado um jogo onde o paciente tem como desafio identificar uma sequência lógica a partir do que é apresentado, sendo trabalhado a memorização e a percepção auditiva. Cabe ressaltar que o jogo apresentado na Figura 1 já estava na biblioteca do JogaVOX e o jogo apresentado na Figura 2 foi utilizado como exemplo do que foi construído para disciplina e aplicado entre os pacientes. 


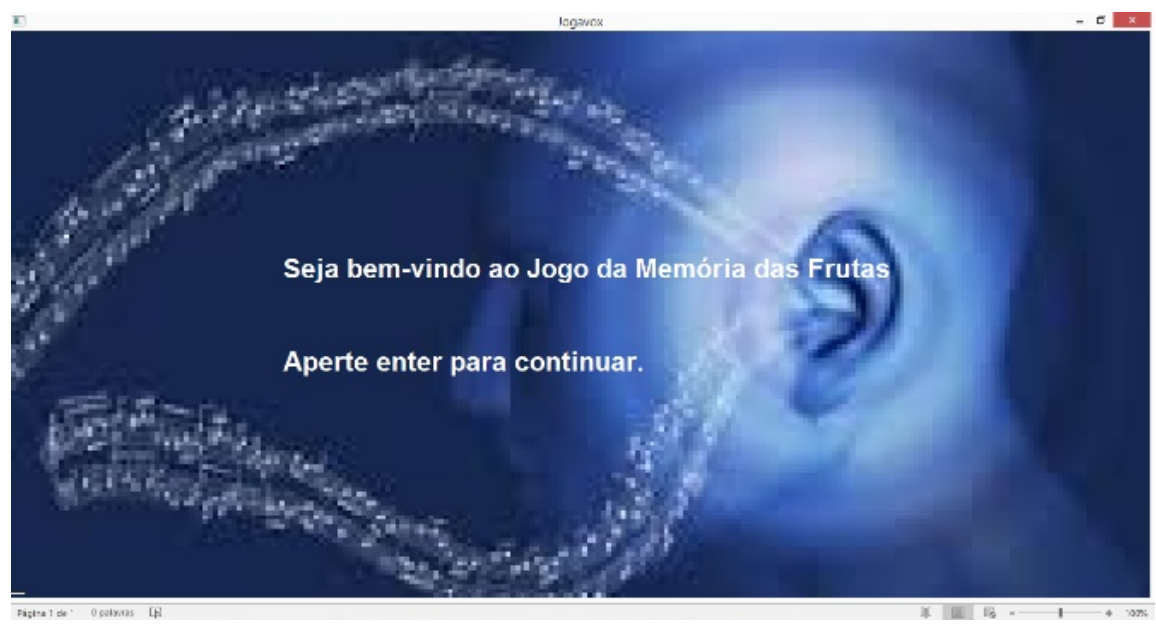

Figura 2.Jogo de Memória das Frutas

\subsection{Teoria do Ator-rede.}

Diante da proposta apresentada podem-se perceber vários atores envolvidos neste cenáriomultidimensional, de cunho profissional e reflexivo. Para tal, foi utilizada a teoria ator-rede, onde (LATOUR,2001) recoloca o lugar da natureza e das coisas, bem como dos humanos e seus artefatos, desfazendo a divisão moderna entre natureza e cultura ou ainda entre sujeito e objeto, reafirmando o lugar das controvérsias no modo de operação de todas as entidades que em algum momento se associam delimitando formas vivas eprontas a se conectarem em novas associações compostas heterogeneamente.

O ator -rede é aquilo que "é induzido a agir por uma vasta rede, em forma de estrela, de mediadores que entram e saem" (LATOUR, 2012, p. 312). Logo, o ator rede é feito para agir,ou seja, para induzir outras entidades a fazerem coisas, através de movimentos edeslocamentos.

Faz-se necessário visualizar simetricamente o contexto sócio-técnico, onde não se trata de separar o conhecimento exato sobre a natureza do exercício do poderentre os homens, mas de seguir a rede que liga constantemente homens e coisas que permite a construção de nosso coletivo. Esta rede não é construída através de discursos ou representação, ela vai além, sendo descortinados através dos objetos que ainda não

encontraram seu lugar ou que simplesmente não possuem lugar nessa divisão tradicional, os híbridos. As redes que o autor nos ensina a seguir são ao mesmo tempo reais como a natureza, narradas como o discurso e coletivas como a sociedade, daí o enredamento para reformulação do pensamento complexo através da vivência social e cotidiana dos diferentes atores envolvidos no processo.

\section{Conclusões}


A utilização do Sistema DOSVOX/JogaVOX possibilitou a criação de jogos compatíveis com às necessidades de tratamento de pacientes com déficits cognitivos relacionados ao $\mathrm{AVC}$, favorecendo a estimulação das capacidades de orientação, atenção, memória, raciocínio, formação de conceitos e solução de problemas.

Os pacientes envolvidos nas referidas atividades relataram satisfação na execução dos jogos apresentados, demonstrando interesse durante as atividades propostas e referindo sentirem-se motivados à realização das mesmas.A avaliação dos resultados permitiu observar que após a aplicação dos jogos construídos no JogaVOX, foram observadas a melhoria das funções cognitivas em geral. A aplicação dos jogos foi bem aceita pelos pacientes durante as diferentes fases do tratamento.

Cabe ressaltar que este trabalho se encontra em processo de construção e que os atores envolvidos no processo continuam em acompanhamento.

\section{Referências:}

BERSCH, R. Tecnologia assistiva e educação inclusiva. In: Ensaios Pedagógicos, Brasília: SEESP/MEC, p. 89 - 94,2006

DA FONSECA, W. de N. T.O uso do software DOSVOX na educação dos deficientes visuais.2012. Especialização ( Mídias na Educação). Universidade Federal do Amapá. 2012. Amapá.

BRASIL. SDHPR -Secretaria Nacional de Promoção dos Direitos da Pessoa com Deficiência $2009 . \quad$ Disponível em: http://www.pessoacomdeficiencia.gov.br/app/publicacoes/tecnologia-assistiva. Acesso em 07 de outubro de 2017.

CUNHA, E.E.-JogaVOX: Ferramenta e Estratégias para Construção de Jogos Educacionais para Deficientes Visuais. 2007, Dissertação (Mestrado em Informática) , Instituto de Matemática, Universidade Federal do Rio de Janeiro, Rio de Janeiro, 2007.

GRIEVE, J.; GNANASEKARAN, L. Neuropsicologia para Terapeutas Ocupacionais -Cognição no Desempenho Ocupacional. Terceira Edição. Editora Santos, São Paulo -SP -2010.

LATOUR, B. Esperança de pandora, Bauru, SP: EDUSC. 2001.

Reagregando o Social. Bauru, SP: EDUSC/ Salvador, BA:EDUFBA. 2012. 
NCE UFRJ. Projeto DOSVOX. Núcleo de Computação Eletrônica da Universidade Federal do Rio de Janeiro. Disponível em: <http://intervox.nce.ufrj.br/dosvox $>$. -Acesso em 07 de outubro de 2017

MORIN, E. Introdução ao pensamento complexo.3. ed. Porto Alegre: Sulina, 2005.

RADABAUGH, M. P. NIDRR'sLong Range PlanTechnology for Access andFunction.ResearchSectionTwo: NIDDR Research Agenda Chapter 5: TECHNOLOGY FOR ACCESS NA D FUNCTION

TROMBLY, C.A.; RADOMSKI, M.V. Terapia Ocupacional para Disfunções Físicas. 5 . Edição. Editora Santos. São Paulo, 2005.http://intervox.nce.ufrj.br/dosvoxacesso em 7 de outubro de 2017. 